Cite this: RSC Adv., 2014, 4, 11050

\title{
On the fate of hydrogen during zirconium oxidation by water: effect of oxygen dissolution in $\alpha-Z r$
}

\author{
Mikaela Lindgren* and Itai Panas
}

Zirconium oxidation by water is accompanied by hydrogen conversion, either $\mathrm{H}_{2}$ is released or hydrogen is picked up by the alloy. Strategies are sought to mitigate the detrimental hydrogen uptake into the metal. The corrosion phenomenon is subdivided into anode and cathode processes caused by electron release upon $\mathrm{O}^{2-}$ oxidation at the metal/oxide interface in the case of the former and electron-proton recombination resulting in hydrogen pick-up or $\mathrm{H}_{2}$ evolution in the case of the latter. In a previous study, the additive dependence of the cathodic hydrogen evolution reaction was analysed. The present study examines the oxygen concentration dependence of the anode potential, presents the impact of oxygen concentration on the co-absorption of hydrogen and merges the anode and cathode processes. The computational model is validated by semi-quantitatively reproducing the experimental solubility limit for oxygen in $\alpha$-Zr. The impact of the emerging conceptual understanding for material development is discussed. Received 2nd January 2014
Accepted 6th February 2014

DOI: $10.1039 / c 4 r a 00020 j$

www.rsc.org/advances the alloy, see e.g. ref. 7-11. In contrast, our effort emphasizes the hydrogen evolution reaction that is the competing pathway to hydrogen pick-up during oxidation of zirconium by water. This implies reformulating the corrosion of the cladding in terms of electrochemistry, i.e. electron release (oxidation) at the anode and electron capture (reduction) at the cathode. The cathode processes comprise hydrogen evolution at oxide grain boundaries or at the oxide/water interface, i.e.

$$
2 \mathrm{e}^{-}+2 \mathrm{H}^{+} \rightarrow \mathrm{H}_{2}
$$

In two recent studies, ${ }^{12,13}$ the electro-catalytic aspect of the cathode process occurring predominantly at inter-grain interfaces between $\mathrm{ZrO}_{2}$ particles was analysed. An alternative cathode process is that hydrogen become dissolved in the oxygen saturated alloy, so-called hydrogen pick-up, according to

$$
\begin{gathered}
2 \mathrm{e}^{-}+2 \mathrm{H}^{+} \rightarrow 2 \mathrm{H} \\
\mathrm{Zr}_{m} \mathrm{O}_{n}+2 \mathrm{H} \rightarrow \mathrm{H}_{2} \mathrm{Zr}_{m} \mathrm{O}_{n} .
\end{gathered}
$$

Yet, irrespective of hydrogen evolution or hydrogen absorption, an electron source is required for both (R1) and (R2a). This anode process occurs at the metal/oxide interface and may be effectively written as

$$
m \mathrm{Zr}+\mathrm{O}^{2-} \rightarrow \mathrm{Zr}_{m} \mathrm{O}+2 \mathrm{e}^{-}
$$

It reflects how the dissolution of oxygen ions in the zirconium alloy implies discharge of electrons to be consumed in (R1) or (R2a).

Given the high solubility of oxygen in $\mathrm{Zr}$, i.e. $\sim 28$ at $\%$ oxygen, ${ }^{\mathbf{1 4}}$ the main objectives of the present study are to
Chalmers University of Technology, Department of Chemical and Biological Engineering, Division of Energy \& Materials, Gothenburg, Sweden. E-mail: mikaela. lindgren@chalmers.se 
determine (1) how the anode potential depends on the oxygen concentration, (2) how the hydrogen solubility in zirconium is affected by the dissolution of oxygen in the metal, and (3) merge the anode and cathode processes to arrive at a comprehensive understanding. The computational approach is validated semi-quantitatively by estimating the experimental solubility limit for oxygen in $\alpha$-Zr. From the fact that zirconia is a large band gap insulator it is inferred that a large overall cell potential will allow the cathode process to take place far from the metal/oxide interface thus increasing the probability for $\mathrm{H}_{2}$ evolution, while the opposite is true for small cell potentials.

\section{Modelling considerations}

The present study constitutes a central part in a comprehensive effort aiming at a detailed mechanistic understanding for the oxidation of zirconium alloys by water. It relies exclusively on atomistic modelling where density functional theory is employed exclusively. The heart of this study is, for different oxygen concentrations, to compute the stabilities of various oxygen distributions in the hexagonal $\alpha$-Zr matrix.

\section{II.A Computational details}

The CASTEP program package ${ }^{\mathbf{1 5}}$ within the Material Studios framework $^{\mathbf{1 6}}$ was utilized and the PBE GGA functional ${ }^{\mathbf{1 7}}$ was employed for all calculations. Core electrons were described by ultrasoft pseudopotentials ${ }^{18}$ in conjunction with $300 \mathrm{eV}$ cut-off energy. $\mathrm{Zr}_{8}$ and $\mathrm{Zr}_{16}$ super-cells of the hexagonal closed packed $\mathrm{Zr}$ metal were employed to investigate the oxygen concentration dependence of the anode potential resulting from oxidation by water. $(2 a, 2 b, c)$ and $(4 a, 2 b, c)$ super-cells of the $\mathrm{Zr}_{2}$ unit cell were considered to obtain the $\mathrm{Zr}_{8}$ and $\mathrm{Zr}_{16}$ host matrices, respectively. The $k$-point sampling of the Brillouin zone was sampled by means of the Monkhorst-Pack scheme. ${ }^{19}$ The $\mathrm{Zr}_{8}$ and $\mathrm{Zr}_{16}$ based super-cells utilized a $4 \times 4 \times 4$ and $2 \times 4 \times 4 k$ points mesh, respectively. Internal consistency of the emerging energetics was checked by reproducing the values obtained for $\mathrm{Zr}_{8} \mathrm{O}, \mathrm{Zr}_{8} \mathrm{O}_{2}, \mathrm{Zr}_{8} \mathrm{O}_{3}$, and $\mathrm{Zr}_{8} \mathrm{O}_{4}$ by the formally equivalent $\mathrm{Zr}_{16} \mathrm{O}_{2}$, $\mathrm{Zr}_{16} \mathrm{O}_{4}$ a $\mathrm{Zr}_{16} \mathrm{O}_{6}$, and $\mathrm{Zr}_{16} \mathrm{O}_{8}$, respectively. Moreover, the oxygen affinity at the low concentration limit was estimated by comparing $\mathrm{Zr}_{16} \mathrm{O}$ to $\mathrm{Zr}_{64} \mathrm{O}$. The latter supercell was obtained by means of a $(4 a, 4 b, 2 c)$ super-cell in conjunction with a $2 \times 2 \times 2$ $k$-points mesh. All results presented include optimization of bond lengths, bond angles, as well as unit cell dimensions and angles.

The present study builds on semi-quantitative arguments to arrive at an internally consistent conceptual understanding. While it does neither include vibrational zero-point corrections nor entropy changes, still it is gratifying to find that the reactivity of $\mathrm{Zr}$ to form $\mathrm{ZrO}_{2}$ by means of $\mathrm{H}_{2} \mathrm{O}$ was found to be only $0.37 \mathrm{eV} / \mathrm{H}_{2} \mathrm{O}$ too small, i.e. $\Delta E(0 \mathrm{~K})=2.72 \mathrm{eV}$ compared to $\Delta G$ $(298 \mathrm{~K})=3.09 \mathrm{eV}$, given the shortcomings of present day density functionals. Finally, it is acknowledged that any successes of studies such as the present rely extensively on the cancellation of errors achieved by making appropriate comparisons.
In order to further assess the findings produced by the PBE GGA functional, comparisons are provided for some structures by means of the PBE0 hybrid functional. ${ }^{20}$ For the PBE0 functional, the norm conserving pseudopotentials ${ }^{21}$ was used in conjunction with a $750 \mathrm{eV}$ cut-off energy for the $\mathrm{Zr}_{8}$ super-cell with $4 \times 4 \times 4 k$-points sampling.

The Linear/Quadratic Synchronous Transit method ${ }^{22}$ was employed to estimate transition states for redistributions of oxygen atoms in the zirconium matrix.

\section{II.B Strategy for sampling structures among $\mathrm{Zr}_{m} \mathrm{O}_{n}$ structures}

There are two types of interstitial sites in the $\mathrm{Zr}$ matrix, octahedral and tetrahedral. Both site types were tested and it was found that octahedral sites are energetically favourable at low concentrations, but a mixed occupancy of both octahedral and tetrahedral sites are preferred at oxygen concentrations in the vicinity of 50 at $\% \mathrm{O}$ and above. In order to obtain candidate octahedral structures, an alternative procedure to that in ref. 23 was employed here. In this study, full configuration sampling in a structural sub-space of the hexagonal $\alpha-\mathrm{Zr}$ host matrix was performed in conjunction with a few complementary candidate structures for interpolation and extrapolation purposes. Thus, all possible permutations of putting one up to eight oxygen atoms in the eight octahedral interstitial sites in the $\mathrm{Zr}_{8}$ structure were evaluated; rotational and reflection symmetry was assumed as well as that the two planes of interstitial sites were identical. Optimized structures for five oxygen concentrations are displayed in Fig. 1, and the sampling procedure for the $\mathrm{Zr}_{8} \mathrm{O}_{1}-\mathrm{Zr}_{8} \mathrm{O}_{8}$ structures can be seen in Fig. 2. The $\mathrm{Zr}_{8} \mathrm{O}_{n}$ structural ensemble was extended to include complementary $\mathrm{Zr}_{16} \mathrm{O}_{n}$ structures. These where related to the $\mathrm{Zr}_{8} \mathrm{O}_{n}$ structures by doubling the unit cell in the $a$ direction and by adding or removing oxygen atoms to obtain oxygen concentrations not accessible with the $\mathrm{Zr}_{8} \mathrm{O}_{n}$ supercell. One $\mathrm{Zr}_{64} \mathrm{O}$ structure was included to address the low oxygen concentration limit. Finally, a suite of structures with different oxygen concentrations from described set was used to study the energy dependence of hydrogen dissolution in these structures.

\section{II.C Rebuilding of electronic structure owing to oxygen dissolution in zirconium}

In order to connect the understanding of oxygen dissolution in zirconium to the familiar nature of the large band gap insulators such as $\mathrm{ZrO}_{2}$, the gradual rebuilding of the density of states as a result of oxygen concentration increase is displayed in Fig. 3. The oxidation can be understood as a titration of electrons at the Fermi energy such that the top of the resulting oxygen associated bands end up $\sim 3-6 \mathrm{eV}$ below the Fermi level $\left(E_{\mathrm{F}}\right)$.

\section{Results and discussion}

The interest in the $\mathrm{Zr}-\mathrm{O}$ phase diagram ${ }^{\mathbf{1 4}}$ extends beyond its relevance to nuclear technology, as its use includes solid oxide 

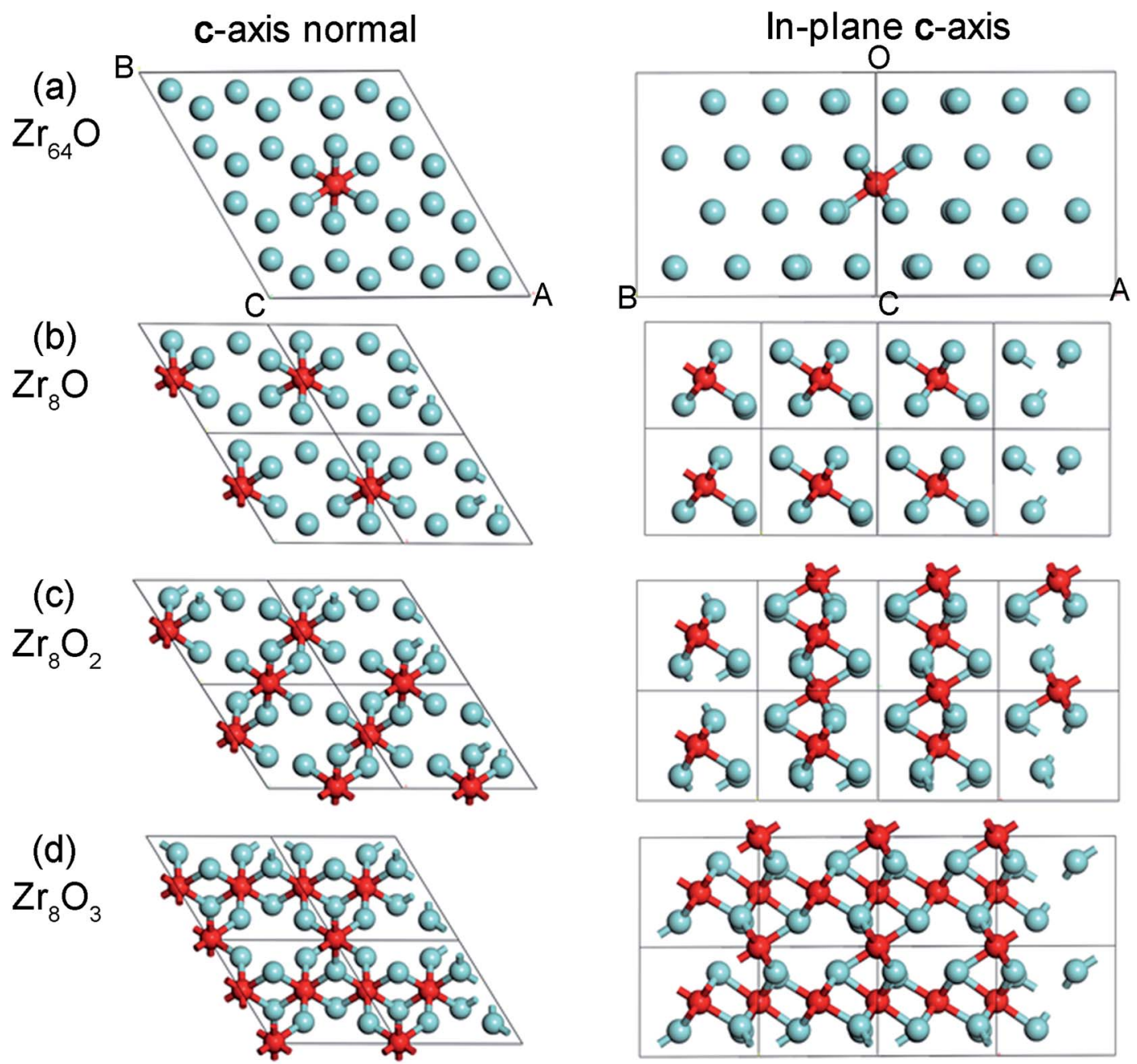

(e)
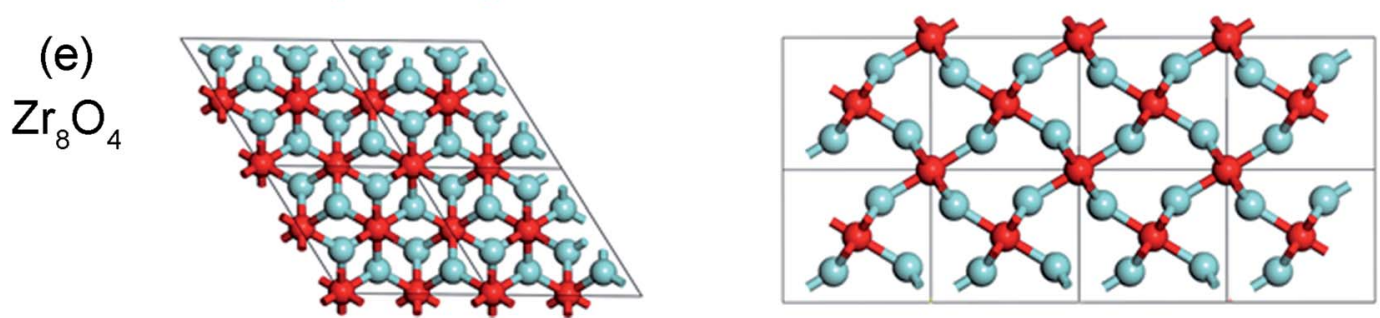

Fig. 1 Optimized zirconium structures with dissolved oxygen for oxygen concentrations (a) 1.5 at\% O, (b) 11 at\% O, (c) 20 at\% O, (d) 28 at\% O, and (e) 33 at\% O. (a) and (b) display $\mathrm{Zr}_{6} \mathrm{O}$ octahedral sites, (c) display chains of edge-sharing octahedra, (d) and (e) constitute sheets of face-sharing octahedra including octahedral.

fuel cell applications as well as thermal barrier coatings. The objective of the present study is to determine how the remarkable solubility of oxygen in hexagonal close-packed zirconium affects the corrosion properties of zirconium alloys. Thus it offers the missing pieces of information to a previous mechanistic investigation on the electro-catalytic additive dependence of the competitiveness of the hydrogen evolution reaction versus hydrogen pick-up in the metal. ${ }^{12,13}$ Indeed, crucial to application is the ability to control the hydrogen evolution reaction, rendering it competitive with the hydrogen pick-up reaction channel. In what follows, the drive for $\mathrm{ZrO}_{2}$ dissolution is quantified from a set of different oxygen distributions, and concentrations and the rates for transitions between metastable structures are estimated to assess their relative importance. Often, the activation energies for structural changes among oxygen distributions are large rendering long lifetimes of meta-stable structures. The examined structures are used to assess how the magnitudes and variations of anode potential change upon increased dissolution of oxygen in zirconium, i.e. if there is an oxygen concentration dependence of the ability of the anode potential to drive the cathode process. In addition, the impact of oxygen concentration on the hydrogen pick-up energetics is addressed. 


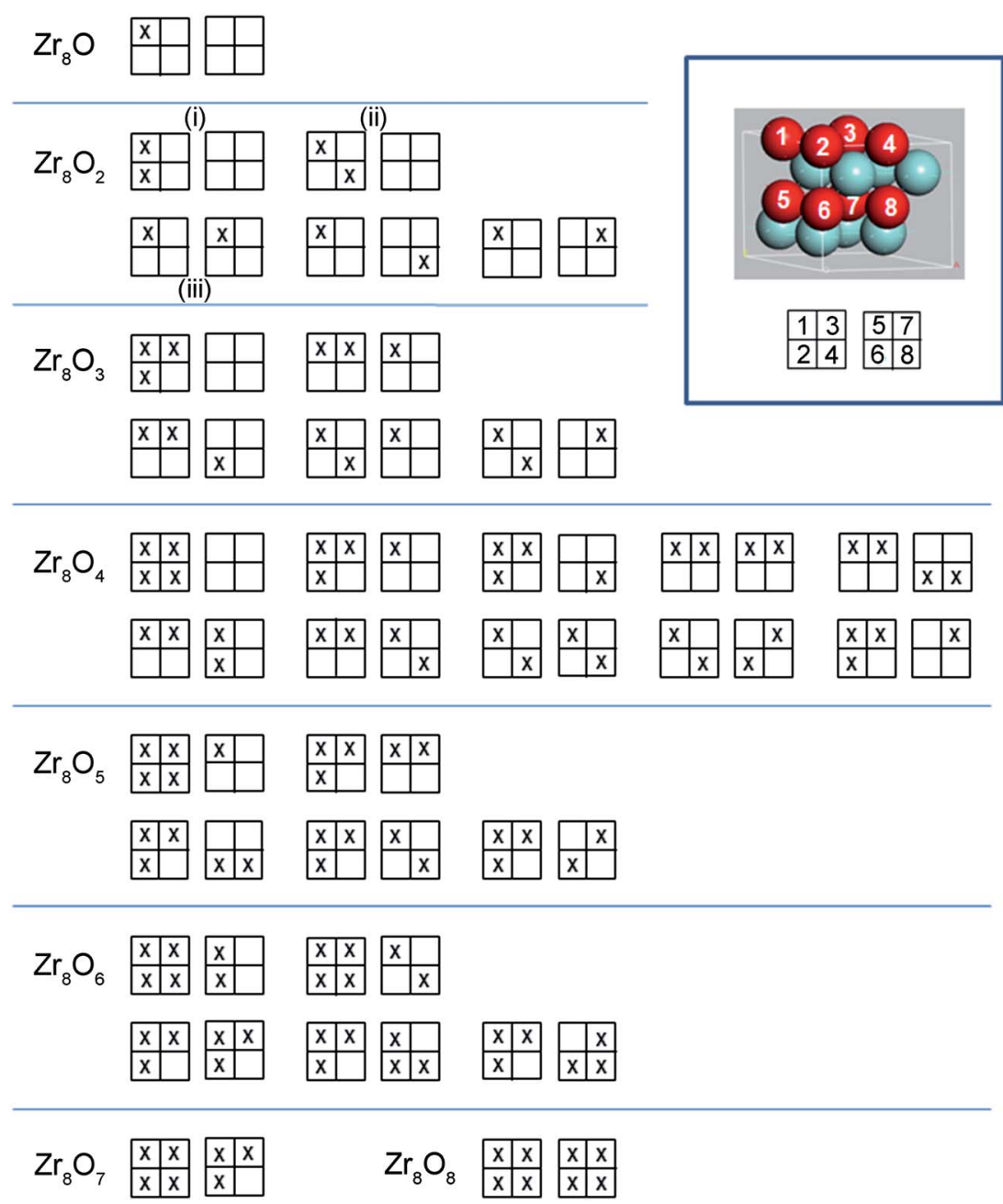

Fig. 2 Sampling procedure for $\mathrm{Zr}_{8} \mathrm{O}_{1}-\mathrm{Zr}_{8} \mathrm{O}_{8}$. All possible permutations to put one up to eight oxygen atoms in the eight octahedral interstitial sites in the $\mathrm{Zr}_{8}$ structure were tested. The most stable structures for each concentration is the top left structure and the stability of the structure is decreasing when going to right. Rotational and reflection symmetry was assumed as well as that the two planes of interstitial sites were identical.

\section{III.A On the dissolution of $\mathrm{m}-\mathrm{ZrO}_{2}$ in $\alpha-\mathrm{Zr}$}

Zirconium is known for its remarkable capacity to dissolve oxygen to the extent that oxygen is considered an alloying element in $\alpha$-Zr. It is interesting to note that while the Zr PDOS at the Fermi level is gradually being depleted, nothing remarkable occurs in the vicinity of $\sim 28$ at $\% 0,{ }^{14}$ see Fig. 3, which is the experimentally reported solubility limit. This suggests that it is the crowdedness of oxygen atoms rather than change in work function, which is decisive for the resulting maximum oxygen solubility in zirconium.

In what follows, the ensemble of structures described in Section II.C, are first ranked with respect to their reaction energies for the reaction

$$
\frac{1}{2} \mathrm{ZrO}_{2}+\left(x-\frac{1}{2}\right) \mathrm{Zr} \rightarrow \mathrm{Zr}_{x} \mathrm{O} .
$$

The numbers obtained by the GGA PBE density functional are displayed in Fig. 4 where the oxygen concentration in at $\%$ is defined as $\frac{1}{1+x} 100 \%$.
It comes out clearly that $\mathrm{ZrO}_{2}$ dissolves in $\mathrm{Zr}(\mathrm{s})$. Moreover, it is noted that for every oxygen concentration, the spread in energy is less that $0.5 \mathrm{eV}$. In order to make any prediction regarding the solubility limit, only the most stable structures, one for each oxygen concentration, are analysed further. These are plotted in the blue curve in Fig. 5. Employing the Boltzmann distribution to the various structures in Fig. 4 does not change the concentration dependence in Fig. 5. Moreover, an overestimate of the configuration entropy as function of oxygen concentration would be to assume degeneracy among configurations, respectively. The stabilities come out comparatively insensitive to the configurational entropy change. In case of $33 \mathrm{at} \% \mathrm{O}$ the shift is $-0.064 \mathrm{eV}$ at $700 \mathrm{~K}$.

In spite of irregularities, the curve clearly displays a minimum. This energy minimum was validated by employ the PBE0 hybrid density functional, which includes $25 \%$ exact exchange, see red curve in Fig. 5. It is tempting to take the crossing of the $E=0 \mathrm{eV}$ line in Fig. 5 to reflect the solubility limit, but this is not done here. Rather, the solubility limit is obtained from the chemical potentials for further $\mathrm{ZrO}_{2}$ 
(a)

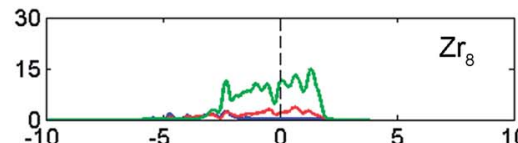

(b)

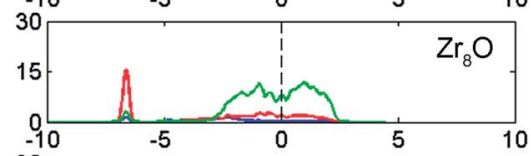

(c)

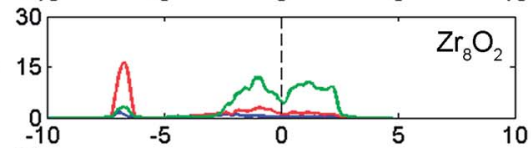

(d)

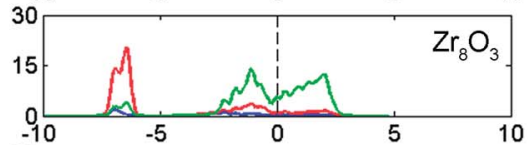

(e)

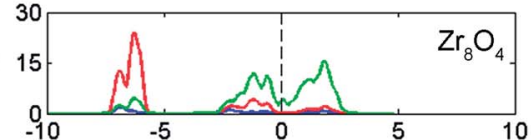

(f)

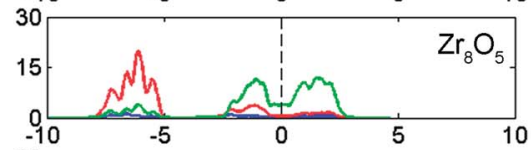

(g)

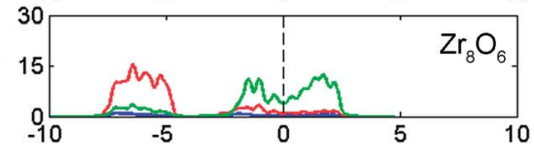

(h)

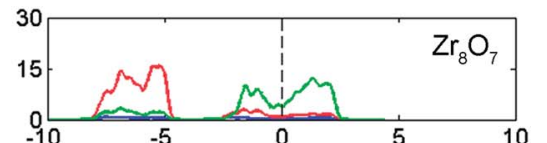

(i)

(j)

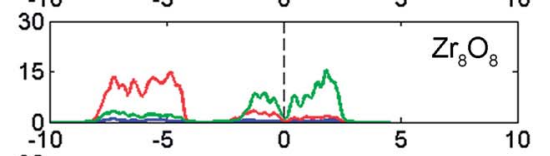

(k)
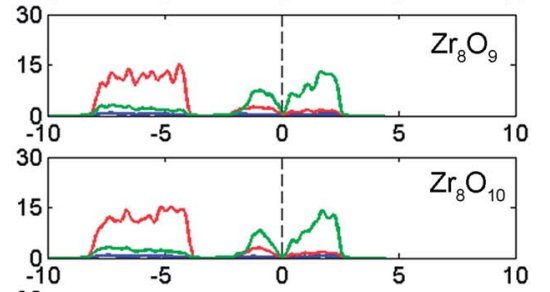

(I)

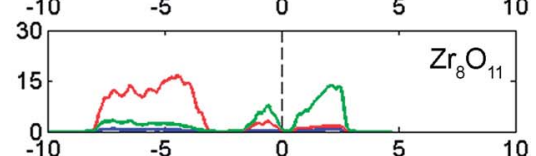

(m)

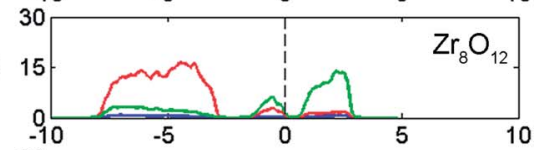

(n)

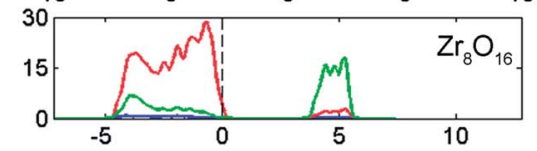

Fig. 3 The rebuilding of the electronic structure owing to increased oxygen concentration in $\alpha$-Zr eventually resulting in a 4 eV band gap of $\mathrm{ZrO}_{2}$; red is PDOS for O $2 \mathrm{p}$, green is PDOS for $\mathrm{Zr} 4 \mathrm{~d}$. Dashed line represents the energy of the highest occupied state in all cases. Vertical axis units are in number of states per eV. Horizontal axis units are in $\mathrm{eV}$.

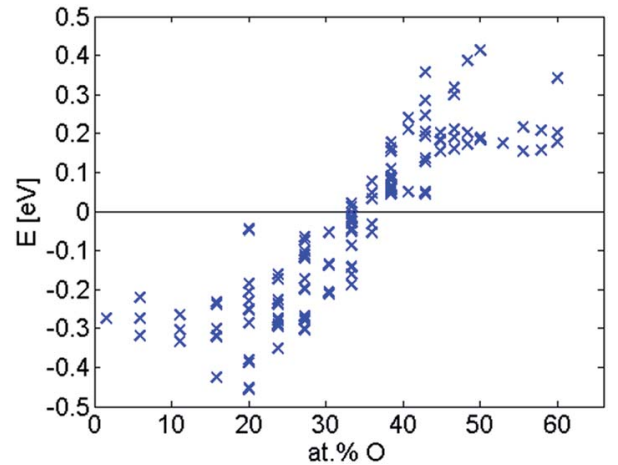

Fig. 4 The energetics of formation of dissolved oxygen structures from $\mathrm{ZrO}_{2}$ and $\mathrm{Zr}$ according to (R4). The spread in energy for a given oxygen concentration comes from different configurations of the oxygen in the Zr matrix.

dissolution in already formed $\mathrm{Zr}_{m} \mathrm{O}_{n}$. This is computed according to

$$
\left(1-\frac{1}{2 m-n}\right) \mathrm{Zr}_{m} \mathrm{O}_{n}+\frac{m}{2 m-n} \mathrm{ZrO}_{2} \rightarrow \mathrm{Zr}_{m} \mathrm{O}_{n+1}
$$

in order to employ the residual drive for dissolution of additional oxygen as a measure of remaining ability to dissolve $\mathrm{ZrO}_{2}$ for each oxygen concentration. The results for $m=8$ and $0 \leq n$ $\leq 8$ are displayed in Fig. 6a for both PBE and PBE0 functionals, while the findings for the extended set including results for $m=$ 16 are displayed in Fig. 6 b.

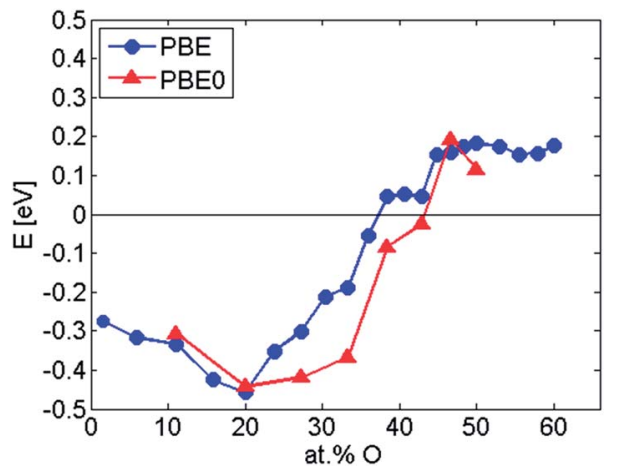

Fig. 5 The energetics of formation of the most stable structures from Fig. 4. Structures calculated with the PBE functional are represented as blue circles and the energetics for some of these structures calculated with the PBEO functional are represented as red triangles.

Support for the existence of the largest drive for $\mathrm{ZrO}_{2}$ dissolution into $\mathrm{Zr}_{m} \mathrm{O}_{n}$ at $\sim 20$ at\% $\mathrm{O}$ is again obtained by comparing PBE and PBE0 results. Besides this global minimum in the oxygen concentration dependent drive for $\mathrm{ZrO}_{2}$ dissolution, spikes emerge in Fig. 6 at concentrations where plateaus are found in Fig. 5. These are taken to reflect transitions between various oxygen orderings in the $\alpha$-Zr matrix. ${ }^{\mathbf{1 4 2 3}}$ It is emphasized that the mere process whereby water oxidizes zirconium alloys tells of a system, which is not in equilibrium. During oxidation, the drive to reach the most stable structure 
(a)

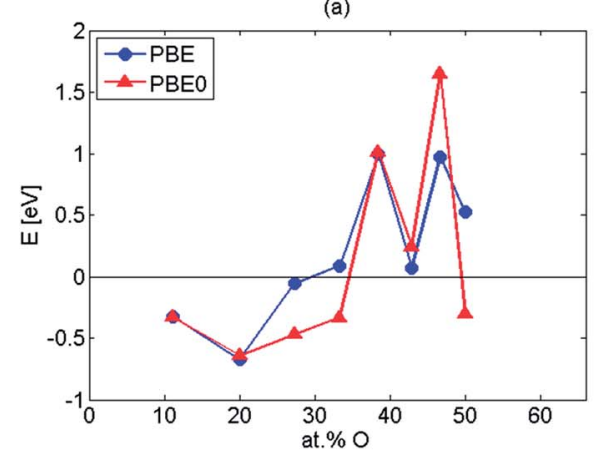

(b)

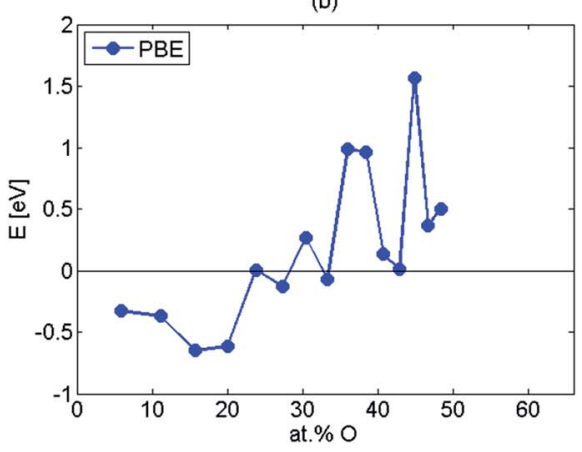

Fig. 6 The chemical potential for $\mathrm{ZrO}_{2}$ dissolution in $\mathrm{Zr}_{m} \mathrm{O}_{n}$ forming $\mathrm{Zr}_{m} \mathrm{O}_{n+1}$ according to (R5) for $m=8$ and $m=16$ in (a) and (b), respectively. Blue circles represent calculations with the PBE functional and red triangles represent calculations with the PBEO functional.

for each local oxygen concentration competes with the continuous inwards dissolution of oxygen into the alloy. For each oxygen concentration, this lends increased importance to the metastable oxygen distributions as compared to the single most stable one. Additional support for this understanding was obtained by calculating activation energies for transitions between typical structures, as summarized in Fig. 1. The obtained numbers, ranging between 0.8 and $2.6 \mathrm{eV}$, represent displacement along the $a$ - or $b$-directions of the former, while activation energies close to the latter are associated with transport along the $c$-direction. The activation barrier of $0.8 \mathrm{eV}$ was calculated between the two $\mathrm{Zr}_{8} \mathrm{O}_{2}$ structures (ii) and (iii) in Fig. 2 and the $2.6 \mathrm{eV}$ barrier was calculated between the (iii) and (i) structures. Repeatedly, when computational data are compared to experiment in the present study, it should be born in mind that periodic boundary conditions have been employed throughout. Yet, both realistic non-equilibrium and equilibrium scenarios exist, which violate the imposed translational symmetry. Hence, segregation of coexisting domains with higher and lower oxygen concentrations is not unexpected to find in systems studied under limited experimental durations when taking into account the large diffusion barriers normal to the basal plane. Neither would spontaneous such symmetry breaking into coexisting sub-micron domains with different oxygen concentrations be unexpected to find at equilibrium. Hence, employing the experimental solubility limit to validate the computational approach is at best suggestive. Having said this, the PBE functional predicts the solubility limit to be at $\sim 29$ at $\% \mathrm{O}$ while the value comes out close to 34 at $\% \mathrm{O}$ in case of PBE0. While it is gratifying to note how these computed numbers come out in agreement with experimental observations, the lower number is commonly reported in the literature, ${ }^{14}$ but the upper one is also found in atom probe studies. ${ }^{13,24}$

\section{III.B Impact of oxygen dissolution in zirconium on the anode potential}

In order to examine the anode potential, $\mathrm{H}_{2}(\mathrm{~g})$, was employed as reference electrode. The oxygen concentration dependent anode potential is estimated according to

$$
\mathrm{Zr}_{m} \mathrm{O}_{n}+\mathrm{H}_{2} \mathrm{O}(\mathrm{g}) \rightarrow \mathrm{Zr}_{m} \mathrm{O}_{n+1}+\mathrm{H}_{2}(\mathrm{~g})
$$

and is depicted in Fig. 7 (blue curve) for the most stable structures for each oxygen concentration.

The residual drive for $\mathrm{ZrO}_{2}$ formation as function of oxygen concentration for these structures is also included in Fig. 7 (red curve). The residual drive is computed from the reaction

$$
\frac{1}{2 m-1} \mathrm{Zr}_{m} \mathrm{O}+\mathrm{H}_{2} \mathrm{O}(\mathrm{g}) \rightarrow \frac{m}{2 m-1} \mathrm{ZrO}_{2}+\mathrm{H}_{2}(\mathrm{~g})
$$

The crossing region in Fig. 7 provides a complementary representation of the solubility limit discussed in Section III.A, where the process beyond the solubility limit corresponds to the disproportionation reaction

$$
\mathrm{Zr}_{m} \mathrm{O}_{n_{\mathrm{s}}+1} \rightarrow\left(1-\frac{1}{2 m-n_{\mathrm{s}}}\right) \mathrm{Zr}_{m} \mathrm{O}_{n_{\mathrm{s}}}+\frac{m}{2 m-n_{\mathrm{s}}} \mathrm{ZrO}_{2}
$$

as long as any $\mathrm{Zr}_{m} \mathrm{O}_{n_{\mathrm{s}}}$ remains. This reaction is the reverse of (R5) but has $\mathrm{Zr}_{m} \mathrm{O}_{n_{\mathrm{s}}}$ as the composition at the solubility limit.

Only the energetics of (R6) and (R7) for the most stable structures are displayed in Fig. 7. However, the activation energies for oxygen diffusion have already added importance to the meta-stable oxygen distributions when addressing the actual corrosion process. Indeed, the energetics for (R6) and (R7) are greatly affected when also including the meta-stable structures, see Fig. 8, which includes all structures in Fig. 2. The

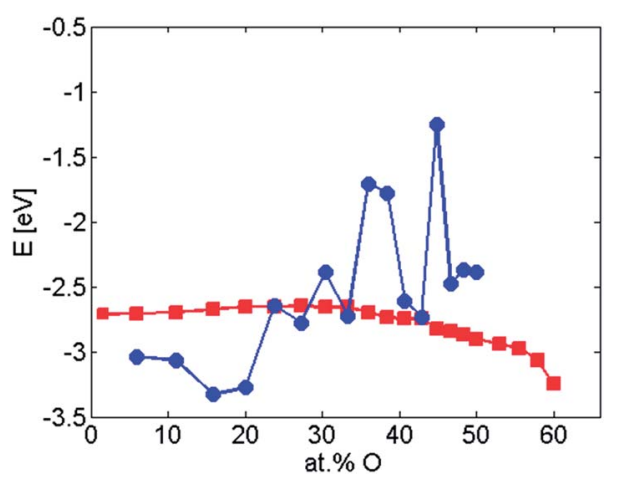

Fig. 7 The oxygen concentration dependent anode potential is represented by the blue circles. The residual drive for the dissolved oxygen structures to form $\mathrm{ZrO}_{2}$ is represented by the red squares. $\mathrm{H}_{2}(\mathrm{~g})$ is the reference electrode for both reactions, see (R6) and (R7). 


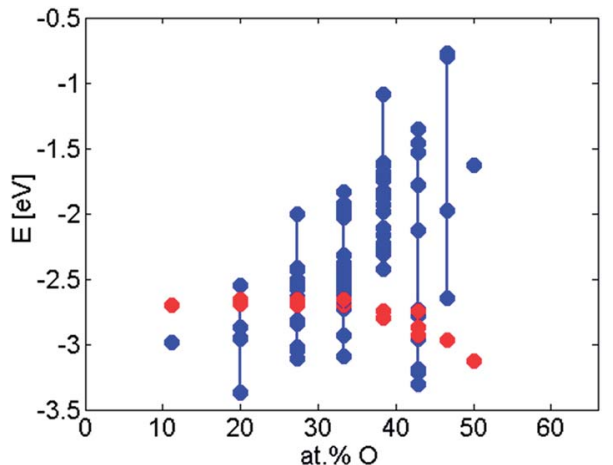

Fig. 8 The energetics of (R6) in blue and (R7) in red for all structures in Fig. 2. The energetics of (R6) is greatly affected by the choice of structures. For some combinations the solubility limit decreases to less than 20 at\% O.

relevance of this finding to the previously discussed cathode process comes out clearly when taking into account that an anode potential of $2.1 \mathrm{eV}$ was required to drive all cathode processes in those studies. ${ }^{12,13}$ For the most stable structures, in the range $20-33$ at $\% \mathrm{O}$, the anode potential is sufficient to drive all cathode processes, see Fig. 7. This is questionable when taking into account the meta-stable structures. In case of the latter, the instantaneous anode potential is no longer expected to comprise a unique number but it comes with a range of locally varying values.

\section{III.C Electronic structure perspectives on the ability of $\mathrm{Zr}_{m} \mathrm{O}_{n}$ to act hydrogen sink}

Oxidation of zirconium by water occurs by inwards oxide growth. The necessary proton reduction may occur either by $\mathrm{H}_{2}$ release or by hydrogen being picked up by the alloy. Mechanisms which lead to $\mathrm{H}_{2}$ release were discussed in previous studies. ${ }^{12,13}$ The reaction, employed to gauge this property here, is

$$
\mathrm{Zr}_{m} \mathrm{O}_{n}+\mathrm{H}_{2}(\mathrm{~g}) \rightarrow \mathrm{H}_{2} \mathrm{Zr}_{m} \mathrm{O}_{n}
$$

The reaction energy for (R9) is plotted as function of oxygen concentration in Fig. 9, where it comes out clearly that the zirconium-oxygen solid solutions act as hydrogen sink versus $\mathrm{H}_{2}(\mathrm{~g})$ well beyond the solubility limit of oxygen.

The drive for hydrogen pick-up is further underscored by the fact that it occurs at the metal/oxide interface where it competes with the $\mathrm{H}_{2}$ formation inside the confining oxide grain boundaries, which introduce an additional $\sim 1.1 \mathrm{eV}$ penalty for the hydrogen evolution due to the confinement. ${ }^{25}$

The impact of co-absorption of oxygen and hydrogen on the electronic structures is displayed in Fig. 10, where the partial densities of states (PDOS) owing to co-absorption of oxygen and hydrogen are shown. The dispersion of the hydrogen associated contributions to the density of states (DOS) is seen to correlate with the edges of the oxygen $2 p$ band, which also envelops the $4 \mathrm{~d}$ contribution to the emerging valence band of monocline $\mathrm{ZrO}_{2}$. It is not unexpected to find the spread in hydrogen affinities in Fig. 9 to be analogous to the spread in anode potentials in Fig. 8, owing to the relationship between the oxygen and hydrogen bands, compare the p-contribution (red: oxygen) in the left panel of Fig. 10, with the s-band (blue) in the PDOS for $2 \mathrm{H}$ in the right panel of Fig. 10.

\section{III.D Impacts of magnitudes and spreads in anode potentials on the cathode process}

In this section, the spreads in anode potentials for three different oxygen concentrations, $c f$. Fig. 8, are merged with the elemental dependence of the energetic for the hydride-proton recombination reactions reported for binary $\mathrm{Zr}$ alloys, ${ }^{\mathbf{1 2 , 1 3}}$ see Fig. 11. Also included in Fig. 11 are the spreads in energies (green band) for the dissolution of $\mathrm{H}_{2}$ corresponding to the different oxygen concentrations, $c f$. Fig. 9.

As the oxygen concentration increases, the spread in anode potentials increases. In as much as these values represent the energy required to form intermediate transition metal hydrides, ${ }^{12,13}$ overlaps between anode potentials and the additive associated curve implies inability to access the hydrogen evolution channel for those elements and potentials.

The energy for $\mathrm{H}_{2}$ dissolution was indeed found to depend on oxygen concentration, yet, the drive for $\mathrm{H}_{2}$ dissolution (a)

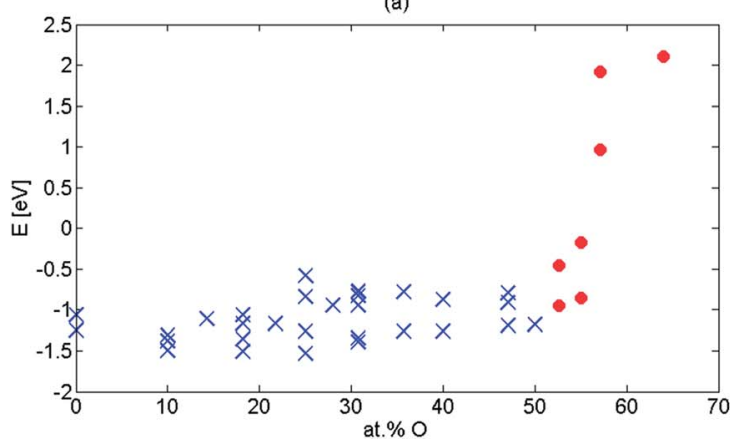

(b)

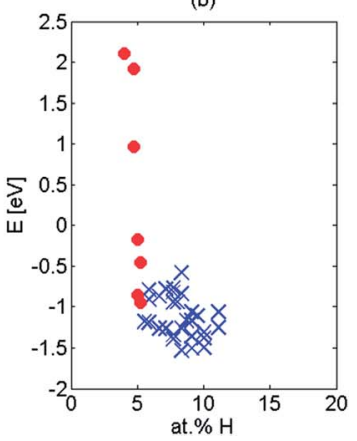

Fig. 9 Energetics for $\mathrm{H}_{2}(\mathrm{~g})$ to be dissolved in various structures with already dissolved oxygen, see (R9). In (a), the oxygen concentrations on the $x$-axis for the structures are before $\mathrm{H}_{2}$ is dissolved in the structures. The hydrogen concentration in (b) is for the structures where $2 \mathrm{H}$ is dissolved. The blue cross indicates oxygen concentrations $\leq 50$ at $\% O$ and the red circles indicates concentrations $>50$ at $\% O$. 
Total DOS

(a)

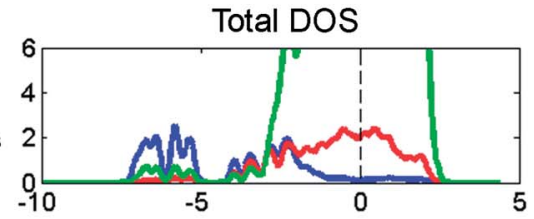

(b)
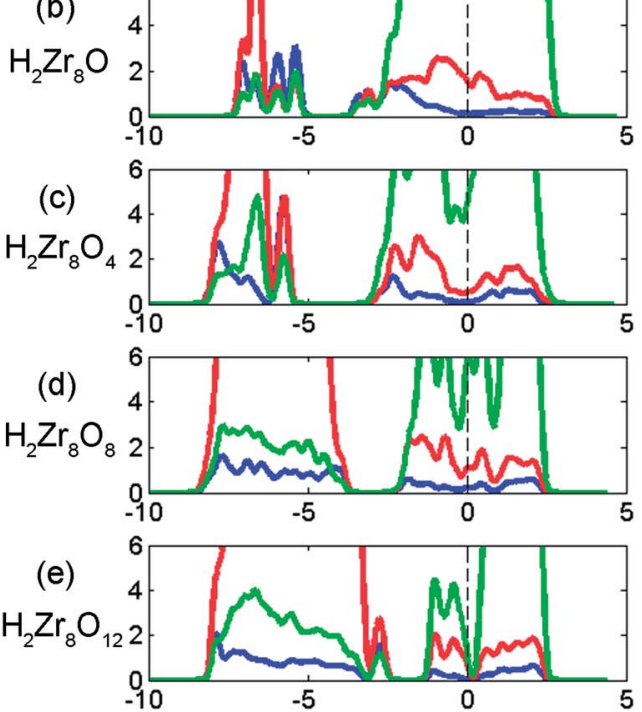

PDOS for $2 \mathrm{H}$
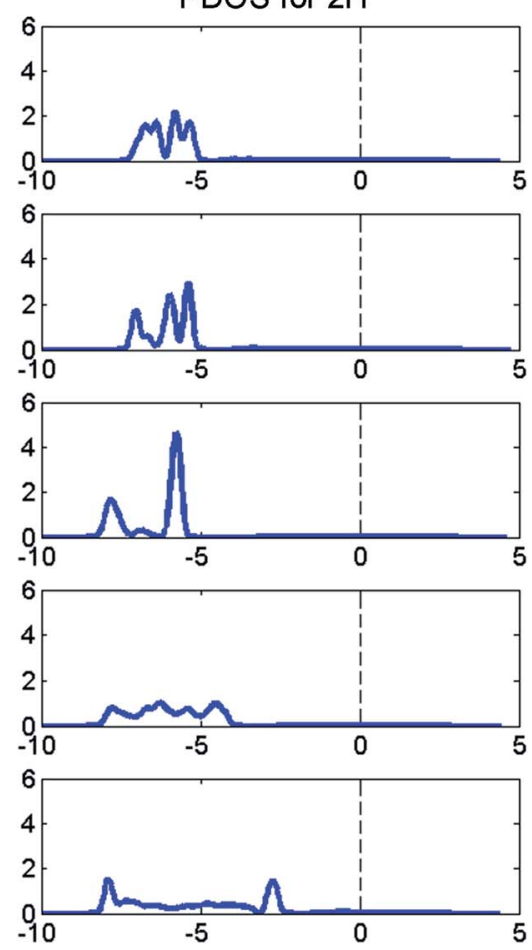

Fig. 10 Influence of hydrogen pick-up on the electronic structure of the zirconium structures with already dissolved oxygen as function of oxygen concentration. The left panel is total DOS and the right panel is the PDOS for the H contribution. Green, red and blue represents d-orbital, $\mathrm{p}$-orbitals and s-orbitals, respectively. Vertical axis units are in number of states per eV. Horizontal axis units are in eV.
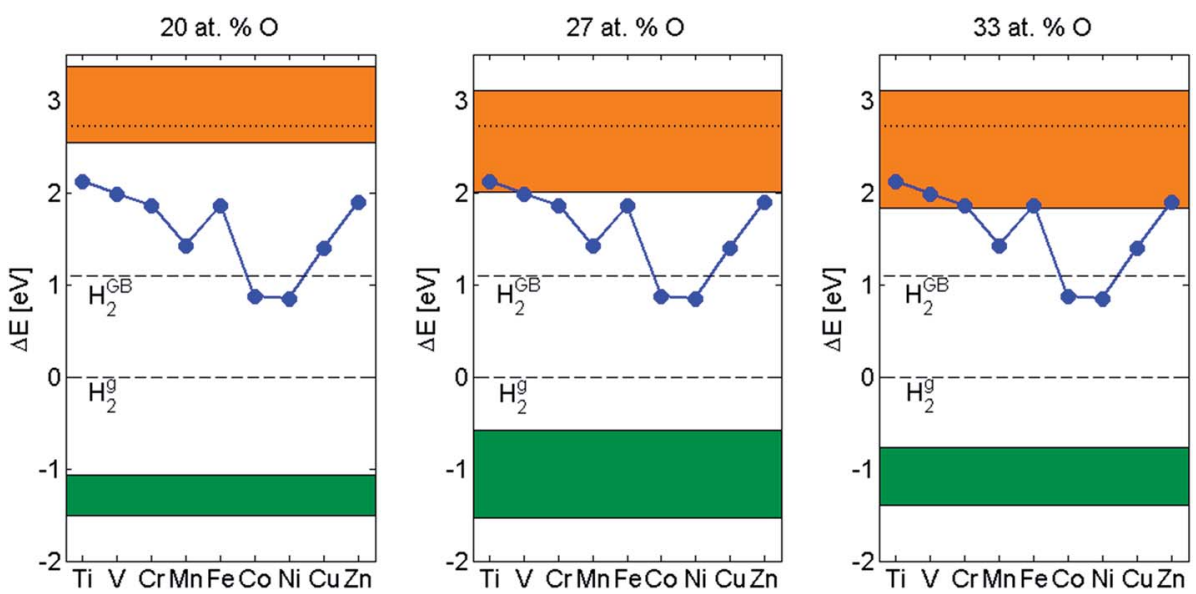

Fig. 11 The blue curve is the elemental dependence on the energetics for the cathode process. ${ }^{12,13}$ The shades area in orange is the spread in anode dependence due to the meta-stable structures in Fig. 3 for 20 at\% O, 27 at\% O and 33 at\% O. The anode potential is not able to drive all cathode reactions in case of 27 at $\% \mathrm{O}$ and 33 at $\%$ O. The shades are in green is the spread in dissolution of $2 \mathrm{H}$ in the some structure with initial oxygen concentrations of 20 at\% O, 27 at\% $\mathrm{O}$ and 33 at\% O. The dashed line at $\mathrm{O} \mathrm{eV}$ corresponds to $\mathrm{H}_{2}(\mathrm{~g})$ and the dashed line at $1.1 \mathrm{eV}$ corresponds to $\mathrm{H}_{2}$ in a oxide grain boundary. ${ }^{25}$

remains large, i.e. in the range $3-4 \mathrm{eV}$, see Fig. 11. It is concluded that the $\mathrm{H}_{2}$ dissolution reaction is significantly less sensitive to the variation among oxygen distributions than the hydrogen evolution reaction in the grain boundary. The latter can by appreciated by considering the distance between the orange ribbons and the dashed line at $1.1 \mathrm{eV}$, the former representing the ranges of anode potentials for different oxygen concentrations while the latter is the energy for $\mathrm{H}_{2}$ release into the grain boundary as compared to free $\mathrm{H}_{2}{ }^{25}$ Hence, the hydrogen pick-up fraction is expected to increase late in the oxidation process, when approaching the so-called pre-transition regime, because the hydrogen evolution process becomes increasingly suppressed. This conclusion is in agreement with what has been observed by Harada et al. ${ }^{26}$ and by Couet et al. ${ }^{27,28}$ 


\section{Concluding remarks}

Because the cell potential for hydrogen evolution during water induced corrosion of $\alpha-\mathrm{Zr}$ is less than that for hydrogen pick-up, avoidance of hydrogen pick-up can only rely on kinetics, i.e. hydride-proton recombination ${ }^{12,13}$ occurring more frequently than hydrogen absorption. Indeed, for all relevant oxygen concentrations, co-absorption of hydrogen is preferred relative to free $\mathrm{H}_{2}$. The findings of the electro-catalytic scenario developed here and in previous studies, ${ }^{\mathbf{1 2}, 13}$ provide clear implications regarding how to mitigate hydrogen uptake: the anode and cathode processes must be separated in space. Whereas the anode process occurs at the metal/oxide interface, it is preferred that the cathode process takes place in the barrier oxide. Improved electron conductivity in the oxide is required in order for the required proton reduction, followed by hydride-proton recombination reaction, to occur further away from the metal/oxide interface. One way to achieve this would be to exploit second-phase particles as electron conductors, since these extend into the oxide and stay metallic as long as contact with the alloy is maintained. A second way is to n-dope $\mathrm{ZrO}_{2}$ by substituting small amounts of $\mathrm{Zr}^{\mathrm{IV}}$ by $\mathrm{Nb}^{\mathrm{IV}}$. Third, would be to improve the electron conductivity in the grain boundaries by additives, which segregate there.$^{29}$ It is cautioned thought, that even if electrons are able to access this cathode site, the residual drive might not suffice to produce $\mathrm{H}_{2}$ in the grain boundary because metastable distributions of oxygen in $\alpha$-Zr may give rise to insufficient local cell potentials. Moreover, it should be born in mind that improved electron conductivity per se, while it reduces the hydrogen pick-up, may cause accelerated zirconium oxidation.

\section{Acknowledgements}

The Swedish Research Council, Westinghouse Electric Sweden, Sandvik Materials Technology, Vattenfall and the EPRI are gratefully acknowledged for financial support.

\section{References}

1 H. G. Rickover, L. D. Geiger and B. Lustman, in US Report, Division of Naval Reactors, Washington, DC, 1975.

2 D. E. Thomas, in Metallurgy of Zirconium, ed. B. Lustman and F. Kerze, McGraw-Hill, New York, 1955.

3 S. Kass, J. Electrochem. Soc., 1960, 107, 594.

4 H. H. Klepfer, J. Nucl. Mater., 1963, 9, 77-84.

5 P. Hohenberg and W. Kohn, Phys. Rev., 1964, 136, B864B871.

6 W. Kohn and L. J. Sham, Phys. Rev., 1965, 140, A1133-A1138.

7 M. V. Glazoff, A. Tokuhiro, S. N. Rashkeev and P. Sabharwall, J. Nucl. Mater., 2014, 444, 65-75.

8 M. Christensen, W. Wolf, C. M. Freeman, E. Wimmer, R. B. Adamson, L. Hallstadius, P. E. Cantonwine and E. V. Mader, J. Nucl. Mater., 2014, 445, 241-250.
9 P. A. Burr, S. T. Murphy, S. C. Lumley, M. R. Wenman and R. W. Grimes, J. Nucl. Mater., 2013, 443, 502506.

10 P. A. Burr, S. T. Murphy, S. C. Lumley, M. R. Wenman and R. W. Grimes, Corros. Sci., 2013, 69, 1-4.

11 S. C. Lumley, S. T. Murphy, P. A. Burr, R. W. Grimes, P. R. Chard-Tuckey and M. R. Wenman, J. Nucl. Mater., 2013, 437, 122-129.

12 M. Lindgren and I. Panas, RSC Adv., 2013, 3, 2161321619.

13 M. Lindgren, G. Sundell, I. Panas, L. Hallstadius, M. Thuvander and $\mathrm{H}$. O. Andrén, in Zirconium in the Nuclear Industry: Seventeenth International Symposium, ed. R. J. Comstock, ASTM, W. Conshohocken, tentatively accepted.

14 J. P. Abriata, J. Garcés and R. Versaci, Bull. Alloy Phase Diagrams, 1986, 7, 116-124.

15 S. J. Clark, M. D. Segall, C. J. Pickard, P. J. Hasnip, M. I. J. Probert, K. Refson and M. C. Payne, Z. Kristallogr., 2005, 220, 567-570.

16 Materials Studio 6.0, Accelrys Inc. p. Simulation software.

17 J. P. Perdew, K. Burke and M. Ernzerhof, Phys. Rev. Lett., 1996, 77, 3865-3868.

18 D. Vanderbilt, Phys. Rev. B: Condens. Matter Mater. Phys., 1990, 41, 7892-7895.

19 H. J. Monkhorst and J. D. Pack, Phys. Rev. B: Solid State, 1976, 13, 5188-5192.

20 C. Adamo and V. Barone, J. Chem. Phys., 1999, 110, 61586170.

21 D. R. Hamann, M. Schlüter and C. Chiang, Phys. Rev. Lett., 1979, 43, 1494-1497.

22 N. Govind, M. Petersen, G. Fitzgerald, D. King-Smith and J. Andzelm, Comput. Mater. Sci., 2003, 28, 250258.

23 B. Puchala and A. Van der Ven, Phys. Rev. B: Condens. Matter Mater. Phys., 2013, 88, 094108.

24 Y. Dong, A. T. Motta and E. A. Marquis, J. Nucl. Mater., 2013, 442, 270-281.

25 M. Lindgren and I. Panas, Confinement Dependence of Electro-Catalysts for Hydrogen Evolution from Water Splitting, Beilstein J. Nanotechnol., accepted.

26 M. Harada and R. Wakamatsu, in Zirconium in the Nuclear Industry: 15th International Symposium, ed. B. Kammenzind and M. Limback, ASTM, W Conshohocken, 2009, pp. 384402.

27 A. Couet, A. T. Motta, R. J. Comstock and R. L. Paul, J. Nucl. Mater., 2012, 425, 211-217.

28 A. Couet, A. T. Motta and R. J. Comstock, Hydrogen Pickup Measurements in Zirconium Alloys: Relation to Oxidation Kinetics, J. Nucl. Mater., accepted.

29 G. Sundell, M. Thuvander and H. O. Andrén, Corros. Sci., 2012, 65, 10-12. 